\title{
Physical Activity and Body-Mass-Index: Do Family, Friends and Teachers Restrain the Risk for Physical Inactivity in Adolescents?
}

\author{
Denise Renninger ${ }^{1, *(\mathbb{D}}$, David Joseph Sturm ${ }^{1} \mathbb{C}$, Adilson Marques ${ }^{2,3} \mathbb{C}^{-}$, Miguel Peralta ${ }^{2,3}$, Stevo Popovic ${ }^{4,5,6}$, \\ Jovan Gardasevic ${ }^{4,6}\left(\mathbb{D}\right.$, Bojan Masanovic ${ }^{4,6,7}$ a and Yolanda Demetriou ${ }^{1}[$
}

1 Department of Sport and Health Sciences, Technical University of Munich, 80992 Munich, Germany; david.sturm@tum.de (D.J.S.); yolanda.demetriou@tum.de (Y.D.)

2 CIPER, Faculty of Human Kinetics, University of Lisbon, 1495-751 Cruz Quebrada, Portugal; amarques@fmh.ulisboa.pt (A.M.); mperalta@fmh.ulisboa.pt (M.P.)

3 ISAMB-Instituto de Saúde Ambiental, University of Lisbon, 1649-026 Lisbon, Portugal

4 Faculty for Sport and Physical Education, University of Montenegro, 81400 Niksic, Montenegro; stevo.popovic@ucg.ac.me (S.P.); jovan@ucg.ac.me (J.G.); bojanma@ucg.ac.me (B.M.)

5 Western Balkan Sport Innovation Lab, 81000 Podgorica, Montenegro

6 Montenegrin Sports Academy, 81000 Podgorica, Montenegro

7 Montenegrosport, 81000 Podgorica, Montenegro

* Correspondence: denise.renninger@tum.de

\section{check for} updates

Citation: Renninger, D.; Sturm, D.J.; Marques, A.; Peralta, M.; Popovic, S.; Gardasevic, J.; Masanovic, B.; Demetriou, Y. Physical Activity and Body-Mass-Index: Do Family, Friends and Teachers Restrain the Risk for Physical Inactivity in Adolescents?. Sustainability 2021, 13, 6992. https:/ / doi.org/10.3390/su13136992

Academic Editor: José Carmelo Adsuar Sala

Received: 1 June 2021

Accepted: 17 June 2021

Published: 22 June 2021

Publisher's Note: MDPI stays neutral with regard to jurisdictional claims in published maps and institutional affiliations.

Copyright: (c) 2021 by the authors. Licensee MDPI, Basel, Switzerland. This article is an open access article distributed under the terms and conditions of the Creative Commons Attribution (CC BY) license (https:/ / creativecommons.org/licenses/by/ $4.0 /)$.

\begin{abstract}
Background: The present study investigates the role of different sources of social support in the relationship of BMI and PA in an international sample of children and adolescents. Methods: Data included 170,211 adolescents (51.5\% female), aged 10-16 $(\mathrm{M}=13.6, \mathrm{SD}=1.64)$ from 37 European countries/regions and were retrieved from the 2013/14 Health Behaviour in School-aged Children (HBSC) international database. Results: PA levels in adolescents are lower for girls, decrease with age, and are lower for overweight and obese adolescents compared to underweight and normal-weight adolescents. Peer support and teacher support significantly predict $\mathrm{PA}\left(\mathrm{OR}_{\text {peer }}=1.11 ; \mathrm{OR}_{\text {teacher }}=0.97\right)$ and vigorous PA frequency $\left(\mathrm{OR}_{\text {peer }}=1.13 ; \mathrm{OR}_{\text {teacher }}=0.94\right)$. Family support only affected vigorous PA frequency $(\mathrm{OR}=1.03)$. Family support had a moderating effect on PA in overweight $\left(\mathrm{OR}_{\mathrm{family}} * \mathrm{BMI}\right.$ (overweight) $\left.=0.94\right)$ and obese $\left(\mathrm{OR}_{\text {family*BMI(obese })}=0.90\right)$ adolescents. Peer support had a moderating effect on vigorous PA frequency in obese $\left(\mathrm{OR}_{\text {peer*BMI(obese })}=1.08\right)$ adolescents. Teacher

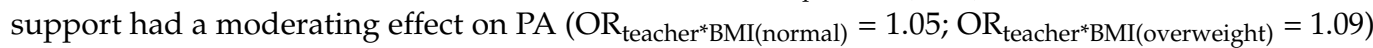
and vigorous PA frequency $\left(\mathrm{OR}_{\text {teacher}}{ }^{\mathrm{BMI}}(\right.$ normal $\left.)=1.07 ; \mathrm{OR}_{\text {teacher*BMI(overweight) }}=1.08\right)$ in normalweight and overweight adolescents. Conclusion: Social support helps adolescents to be active. Especially for obese adolescents, support by and relations with peers are important to reach a significant amount of PA.
\end{abstract}

Keywords: social support; peers; HBSC; secondary analysis; overweight; obesity; adolescents

\section{Introduction}

Physical activity (PA) in childhood and adolescence is associated with several beneficial health outcomes [1]. Overall, evidence shows consistent positive effects of total PA on adiposity, multiple cardio metabolic biomarkers, physical fitness and bone health [2]. Some support can be observed of a positive relationship between total PA and quality of life and well-being, psychological distress and motor skill development [2]. Additionally, Marques and colleagues observed, that in an international sample of 167,021 children and adolescents aged 10-16 years, those who engaged in daily PA reported greater subjective health in measures like headaches, stomachaches, backache, dizziness, feeling low, irritability, nervousness, and sleep difficulties [3]. Generally, longer activities with higher intensity can provide greater health benefits, but at the same time, even the smallest increase in PA can offer health improvements in high-risk groups such as obese children [2,4]. Usually, serious health conditions manifest themselves in adulthood, but studies show that 
childhood PA behavior is largely stable over the entire lifespan and thus determines later health outcomes $[5,6]$. As PA contributes directly and indirectly to acute and long-term health consequences, it is important to achieve sufficient levels of PA during childhood and adolescence. Therefore, the World Health Organization (WHO) recommends children and adolescents perform an average of $60 \mathrm{~min}$ per day of moderate-to-vigorous-intensity PA (MVPA) during the week [1]. Nevertheless, data from the 2014 HBSC study demonstrate insufficient levels of MVPA in $75 \%$ of boys and $85 \%$ of girls across the WHO European region. Further, the data show significantly lower levels of MVPA in girls across all age groups and a general decrease in MVPA with increasing age [7].

At the same time, the global prevalence of overweight and obesity in children and adolescents has continuously increased over the last years and has become a major public health challenge of the 21st century [7,8]. From 1975 to 2016, the global age-standardized prevalence of obesity increased from $0.7 \%$ to $5.6 \%$ in girls and from $0.9 \%$ to $7.8 \%$ in boys resulting in about 50 million girls and 74 million boys with obesity worldwide. Additionally, about 213 million children and adolescents were overweight in 2016 [8].

Evidence from cross-sectional studies reports an inverse relation between PA and body fat in children and adolescents [9-11]. For example, the European Youth Heart Study, conducted among children aged 9-10 years, found an independent and inverse association of both time spent at MVPA and time spent performing vigorous PA with body fatness [10]. A study on Hispanic children and adolescents aged 4-19 years in the USA also reports that PA levels were influenced by age, gender and body mass index (BMI). Thereby, total PA was lower in the overweight sample compared to non-overweight peers [11]. Likewise, systematic reviews support this association. A systematic review found obese children and adolescents engaged in less than 60 min per day and spent significantly less time in MVPA compared with non-obese controls [12]. Another review examining associations between objectively measured habitual PA and adiposity in children and adolescents showed consistent evidence of negative associations between PA and adiposity [13]. Considering the health consequences caused by overweight and obesity [14], a lack of PA in overweight and obese youth is even more alarming. Therefore, determining possible supportive factors for PA, that reduce the negative influence of overweight or obesity seems important.

The ecological model of four domains of active living proposes factors of the policy, informational, social-cultural, and natural environment that influence an active lifestyle [15]. Among other influences, this model suggests that intra-individual and social environmental variables interact to promote health behaviors. In this sense, social support is one major factor influencing PA and can be received from various sources. Reimers and colleagues exhibited associations between social support and modeling from family and peers with all kinds of PA participation [16]. Baumann and colleagues identified in their systematic review family support as a correlate of both children's and adolescents' PA [17]. Whereas Craggs and colleagues could not confirm associations between children's or adolescents' PA with family support or peer support, but general social support determined PA in adolescents aged 14 years and older [18]. A study from Duncan and colleagues indicated a positive relationship of social support with youth PA. Among the three studied sources of support (family, siblings and friends), support from friends had the strongest impact on PA [19]. This is in line with findings from Sallis and colleagues, who reported a significant positive influence of peer support on vigorous PA of boys and girls [20]. The review from Van der Horst and colleagues builds on these findings and extends evidence for a positive association between parental support with children's PA and a favorable correlation of adolescents' PA with friend support [21]. Badura and colleagues assessed social support from family and peers as a confounder in the relationship between organized leisure time activities and well-being. However, the actual influence of these sources of social support on PA remains unexplored [22]. Recent research from Gill and colleagues found support from family and friends to be a strong predictor for meeting daily and weekly PA recommendations and participating in sports teams [23]. Summarizing the mentioned evidence, adolescents can receive support from different sources. Support from family and 
friends is well researched and it seems that peer support has the most valuable effect on the accumulation of PA in adolescence.

Drawing the attention back to the inverse relationship between high BMI with PA, the question arises whether social support can affect this association in a favorable way. To our knowledge, evidence on this topic is scarce. One study from Belgium examined differences in psychosocial correlates of PA between normal-weight and overweight adolescents aged 11-19 years [24]. Results revealed significantly lower intensities of PA in overweight adolescents than in their normal-weight peers. Yet, associations between psychosocial variables (i.e., social support) and total PA were similar in overweight and normal-weight adolescents. Among other psychosocial factors, more social support from family and friends indicated higher levels of PA independent of weight status [24]. Another crosssectional study from the United States investigated the influence of weight status on social-ecological correlates of PA in adults. Likewise, normal-weight individuals reported significantly higher amounts of PA than overweight individuals, who in turn reported significantly higher amounts of PA than obese participants. In addition, social support was found to be positively associated with PA across all weight groups [25]. In general, the association between weight and social support seems inconsistent [23].

Considering the lack of evidence, this study aims to examine the role of different sources of social support in the relationship of BMI and PA in an international sample of children and adolescents. We aim to include the sources of social support from family and friends, as literature identifies these two as a relevant influence on PA. Additionally, support from teachers will be included in the analysis because children and adolescents spend most of their waking hours in school and teachers might have a huge influence on them. With this investigation we want to extend the knowledge on how different sources of support affect adolescents' PA behavior and whether this varies in adolescents of different BMI status.

\section{Materials and Methods}

\subsection{Study Population}

Data were retrieved from the 2013/14 Health Behaviour in School-aged Children (HBSC) international database. The HBSC is a cross-national survey and included 43 nations in 2013/14. Details on the methods and design of the HBSC are described elsewhere [26]. The survey was conducted by the ethical guidelines from ethical committees from each country. School administrators in each country gave their consent and legal guardians gave written informed consent. Participation was anonymous and the administration of the surveys was conducted according to the HBSC standard survey protocol [26]. The target population of the HBSC study were children and adolescents aged 10 to 16, attending school regularly. The HBSC 2014 survey sample comprised 214,080 students. For the present study, we selected students who reported physical activity levels, exercise frequency and social support variables, which resulted in a final sample size of 170,211 adolescents $(51.5 \%$ female), aged 10-16 ( $\mathrm{M}=13.6, \mathrm{SD}=1.64)$ from 37 European countries and regions (Albania, Austria, Belgium, Bulgaria, Croatia, Czech Republic, England, Estonia, Finland, France, Germany, Greece, Greenland, Hungary, Iceland, Ireland, Israel, Italy, Latvia, Luxembourg, Macedonia, Malta, Republic of Moldova, Netherlands, Norway, Poland, Portugal, Romania, Russia, Scotland, Slovakia, Slovenia, Spain, Sweden, Switzerland, Ukraine and Wales). Data from Armenia, Canada and Denmark were excluded due to unusually high missing data in the requested variables.

\subsection{Measures}

\subsubsection{Sociodemographic Characteristics}

Participants reported age, gender, and answered six items of the family affluence scale (FAS) [27], which serves as a valid proxy for socioeconomic status (SES). The sum score of the FAS was calculated and categorized into three categories of low $(0-20 \%)$, medium $(21-80 \%)$, and high family affluence $(81-100 \%)$. 


\subsubsection{Body Mass Index}

Height and body weight were assessed by self-reports. The BMI categories (underweight, normal, overweight, obese) were determined by the age- and gender-related thresholds regarding the 15th, 85th and 97th percentiles [28,29].

\subsubsection{Social Support}

Social support from family and peers was measured by four items each from the MSPSS scale [30]. Participants answered the questions on a 7-point Likert scale from very strongly disagree (1) to very strongly agree (7). Another three items measured teacher support on a 5-point Likert scale from strongly disagree (1) to strongly agree (5). The mean scores were computed and standardized; higher scores indicate higher perceived social support.

\subsubsection{Physical Activity}

Adolescents rated the number of days over the past week that they were physically active, for a total of at least $60 \mathrm{~min}$ per day. Answers were given on an 8-point scale $(1=0$ days to $8=7$ days $)$. Responses were dichotomized into $\leq 6$ days and 7 days, according to the physical activity guidelines in 2014 [31].

According to the WHO recommendation to incorporate vigorous-intensity aerobic activities, as well as those that strengthen muscle and bone, at least 3 days a week [31], adolescents reported the weekly frequency of vigorous PA by answering the question: "Outside school hours: how often do you usually exercise in your free time so much that you get out of breath or sweat?" on an 8 -point scale $(1=$ never to $8=$ every day $)$. Responses were dichotomized into $<2-3$ times per week and $\geq 2-3$ times per week.

\subsection{Data Analysis}

We performed a series of linear and logistic regression models accounting for the random effect of schools and countries using the lme4 package in R. Initially, the variability at the country and the school level was assessed. Then, we analyzed the associations between social support variables and age, sex, BMI, and SES. In the next step, we assessed the strength and significance of the social support by family members, peers, and teachers with PA and vigorous PA frequency. Lastly, an interaction term was included, which considered social support with each of the BMI levels, to analyze their moderating role in the association of the perceived family, peer, and teacher support. All models were adjusted for sex, age, and SES.

\section{Results}

The data reveal, that every fifth adolescent $(20.0 \%)$ in Europe aged from $10-16$ years meets the WHO PA guideline. Almost three-quarters of adolescents $(72.7 \%)$ engage more than twice per week in vigorous PA. Further descriptive data can be found in Supplementary Table S1, which displays the sociodemographic characteristics of the sample, the BMI, and the perceived levels of social support by family, friends and teachers.

The prevalence of meeting the PA and vigorous PA frequency guideline varies significantly across age, BMI and SES categories as well as across countries (see Supplementary Tables S2-S5). The prevalence for PA was higher for boys in all countries, except in Moldova, where differences were not significant. Only in Finland, Iceland and Norway, the proportion of girls meeting the vigorous PA frequency guideline was not significantly different from the proportion of boys (see Supplementary Table S2).

Supplementary Table S3 displays the decreasing prevalence of PA and vigorous PA frequency with increasing age across the sample with the exceptions of Greenland and Moldova. The trend was evident in both boys and girls: with increasing age, the prevalence of meeting the PA guidelines decreased for boys (the prevalence for 11-year-olds was $30.1 \%$ and $20.4 \%$ for 15 -year-olds) and girls ( $21.0 \%$ in 11 -year olds; $10.7 \%$ in 15 -year-olds). We observed the same pattern for the vigorous PA frequency (81.5\% in 11-year-old boys and 
$75.6 \%$ in 15 -year-olds, while 11-year old girls' prevalence was $74.5 \%$ and 15 -year-olds was $59.6 \%)$.

With only a few country-specific exceptions, the prevalence of meeting the PA guideline was lower for overweight and obese adolescents compared to normal and underweight adolescents. With regard to the vigorous PA frequency this BMI-related pattern holds untrue because in 12 countries overweight and/or obese populations have a higher prevalence of meeting the guideline (see Supplementary Table S4). A consistent finding was that adolescents with a higher SES score had a higher prevalence of meeting the PA guideline (see Supplementary Table S5).

The associations between the social support by family, friends and teachers with the demographic and socioeconomic variables are displayed in Table 1. Boys perceive a slightly higher family support $(\beta=0.05)$ and less peer support $(\beta=-0.40)$ than girls, while the sex-related difference in teacher support is small $(\beta=-0.02)$. With increasing age, the perceived support from the family decreases while the teacher support increases. Compared to the age group of the 11 year-olds, peer support significantly decreases by the age of 13 but not within the group of 15 year-olds. We observed a socioeconomic gradient for the family and peer support; adolescents with higher SES perceive higher family ( $\beta=0.17$ to 0.24 ) and peer support ( $\beta=0.16$ to 0.26 ), while the support by teachers is rather independent of the SES ( $\beta=-0.01$ to 0.02 ). A higher BMI score is associated with lower family ( $\beta$ ranging from -0.03 to -0.19 ) and peer support $(\beta=-0.11$ to -0.22 ), but teacher support is similar ( $\beta=0.02$ to 0.03 ) across BMI categories.

Table 1. Multivariate associations of demographic, social and socioeconomic factors with social support by family, peers and teachers based on three-level linear regression models.

\begin{tabular}{|c|c|c|c|c|c|c|}
\hline & \multicolumn{2}{|c|}{ Family Support } & \multicolumn{2}{|c|}{ Peer Support } & \multicolumn{2}{|c|}{ Teacher Support } \\
\hline & $\beta$ & $95 \% \mathrm{CI}$ & $\beta$ & $95 \% \mathrm{CI}$ & $\beta$ & $95 \% \mathrm{CI}$ \\
\hline \multicolumn{7}{|l|}{ Fixed effects } \\
\hline Intercept & 5.88 & {$[5.75,6.00]$} & 5.54 & {$[5.43,5.65]$} & 1.82 & {$[1.76,1.89]$} \\
\hline \multicolumn{7}{|l|}{ Sex (ref. = Female) } \\
\hline Male & 0.05 & {$[0.03,0.07]$} & -0.40 & {$[-0.42,-0.38]$} & -0.02 & {$[-0.03,-0.01]$} \\
\hline \multicolumn{7}{|l|}{ Age (ref. = 11 years) } \\
\hline 13 years & -0.31 & {$[-0.33,-0.29]$} & -0.04 & {$[-0.06,-0.02]$} & 0.39 & {$[0.38,0.40]$} \\
\hline 15 years & -0.47 & {$[-0.49,-0.45]$} & -0.02 & {$[-0.05,0.001]$} & 0.55 & {$[0.53,0.56]$} \\
\hline \multicolumn{7}{|c|}{ Relative SES (ref. = Low) } \\
\hline Medium SES & 0.17 & {$[0.15,0.20]$} & 0.16 & {$[0.13,0.18]$} & -0.01 & {$[-0.02,0.01]$} \\
\hline High SES & 0.24 & {$[0.21,0.27]$} & 0.26 & {$[0.23,0.29]$} & 0.02 & {$[0.004,0.04]$} \\
\hline \multicolumn{7}{|c|}{ Relative BMI (ref. = Underweight) } \\
\hline Normal weight & -0.03 & {$[-0.05,-0.01]$} & 0.001 & {$[-0.02,0.03]$} & 0.02 & {$[0.01,0.05]$} \\
\hline Overweight & -0.11 & {$[-0.14,-0.08]$} & -0.11 & {$[-0.14,-0.08]$} & 0.03 & {$[0.02,0.05]$} \\
\hline Obese & -0.19 & {$[-0.23,-0.15]$} & -0.22 & {$[-0.27,-0.18]$} & 0.02 & {$[0.00,0.05]$} \\
\hline Random effects & \multicolumn{2}{|c|}{ Variance (SD) } & \multicolumn{2}{|c|}{ Variance (SD) } & \multicolumn{2}{|c|}{ Variance (SD) } \\
\hline Country & \multicolumn{2}{|c|}{$0.15(0.38)$} & \multicolumn{2}{|c|}{$0.11(0.33)$} & \multicolumn{2}{|c|}{$0.04(0.19)$} \\
\hline School & \multicolumn{2}{|c|}{$0.04(0.21)$} & \multicolumn{2}{|c|}{$0.04(0.19)$} & \multicolumn{2}{|c|}{$0.04(0.21)$} \\
\hline
\end{tabular}

$n=134,148$ from 37 countries from the 2013/2014 HBSC survey. Data are beta coefficients $(\beta)$ and $95 \%$ confidence intervals. Statistically significant values are indicated in bold. $\mathrm{CI}=$ confidence interval; $\mathrm{SES}=$ socioeconomic status; ref. = reference category; $\mathrm{SD}=$ standard deviation; HBSC $=$ Health Behaviour in School-aged Children .

Table 2 displays the associations between the social support variables and the physical activity outcomes. Family support does not significantly increase the odds to perform at least 60 min MVPA per day, but slightly increases the odds to engage more than 2-3 times per week in vigorous activities during leisure time. Peer support is associated with higher odds of meeting the PA $(\mathrm{OR}=1.11)$ and vigorous PA frequency guideline $(\mathrm{OR}=1.13)$ and teacher support is associated with lower odds of PA (0.97) and vigorous PA frequency $(\mathrm{OR}=0.94)$. 
Table 2. Associations between social support by family, peers and teachers and physical activity outcomes. Results from three-level logistic regression models with random effects adjusted for BMI, age, sex, and SES.

\begin{tabular}{|c|c|c|c|c|}
\hline & \multicolumn{2}{|c|}{ Meet PA Guideline } & \multicolumn{2}{|c|}{$\begin{array}{c}\text { Meet Vigorous PA Frequency } \\
\text { Guidelines }\end{array}$} \\
\hline & OR & $95 \% \mathrm{CI}$ & OR & $95 \% \mathrm{CI}$ \\
\hline \multicolumn{5}{|l|}{ Fixed effects } \\
\hline Family support & 1.02 & {$[1.00,1.03]$} & 1.03 & {$[1.01,1.05]$} \\
\hline Peer support & 1.11 & {$[1.09,1.12]$} & 1.13 & {$[1.11,1.15]$} \\
\hline Teacher support & 0.97 & {$[0.96,0.99]$} & 0.94 & {$[0.93,0.96]$} \\
\hline Random effects & \multicolumn{2}{|c|}{ Variance (SD) } & \multicolumn{2}{|c|}{ Variance (SD) } \\
\hline Country & \multicolumn{2}{|c|}{$0.14(0.37)$} & \multicolumn{2}{|c|}{$0.12(0.34)$} \\
\hline School & \multicolumn{2}{|c|}{$0.13(0.37)$} & \multicolumn{2}{|c|}{$0.08(0.29)$} \\
\hline
\end{tabular}

$n=134,148$ from 6560 schools from 37 countries from the 2013/2014 HBSC survey. Statistically significant values are indicated in bold. $\mathrm{PA}=$ physical activity; $\mathrm{OR}=$ odds ratio; $\mathrm{CI}=$ confidence interval; $\mathrm{SD}=$ standard deviation.

We further tested the associations of social support and physical activity behavior across BMI categories, thus included interaction terms in the fully adjusted models (see Tables 3-5). Family support significantly moderates the association between PA and BMI in overweight and obese adolescents compared to underweight and normal-weight adolescents. However, it does not moderate the relationship of vigorous PA frequency per week and BMI. Social support from peers does not moderate the relationship between BMI and physical activity or vigorous PA frequency. One exception is the interaction of peer support and obesity, which significantly increases the odds for vigorous PA frequency. Teacher support moderates the relationship between PA and BMI as well as the relationship between vigorous PA frequency and BMI, both in the group of normal-weight and overweight adolescents. In underweight and obese adolescents, a higher teacher support is associated with lower odds of PA and vigorous PA frequency, while the odds for normal and overweight adolescents slightly increase.

Table 3. Association between family support and selected physical activity indicators: Results from adjusted three-level logistic regression models with mixed effects; including the family support $x$ BMI interaction term.

\begin{tabular}{|c|c|c|c|c|}
\hline & \multicolumn{2}{|c|}{ Meet PA Guideline } & \multicolumn{2}{|c|}{$\begin{array}{c}\text { Meet Vigorous PA Frequency } \\
\text { Guidelines }\end{array}$} \\
\hline & OR & $95 \% \mathrm{CI}$ & OR & $95 \% \mathrm{CI}$ \\
\hline \multicolumn{5}{|l|}{ Main effects } \\
\hline Family support & 1.10 & {$[1.06,1.14]$} & 1.11 & {$[1.07,1.14]$} \\
\hline \multicolumn{5}{|l|}{ BMI (ref. = Underweight) } \\
\hline Normal weight & 0.96 & {$[0.93,1.00]$} & 1.22 & {$[1.18,1.26]$} \\
\hline Overweight & 0.72 & {$[0.69,0.76]$} & 1.02 & {$[0.97,1.06]$} \\
\hline Obese & 0.56 & {$[0.52,0.61]$} & 0.79 & {$[0.75,0.84]$} \\
\hline \multicolumn{5}{|l|}{ Interaction effects } \\
\hline $\begin{array}{l}\text { Family support * BMI } \\
\text { (Normal weight) }\end{array}$ & 0.97 & {$[0.94,1.01]$} & 0.99 & {$[0.96,1.02]$} \\
\hline $\begin{array}{c}\text { Family support * BMI } \\
\text { (Overweight) }\end{array}$ & 0.94 & {$[0.89,0.99]$} & 0.98 & {$[0.94,1.03]$} \\
\hline $\begin{array}{l}\text { Family support * BMI } \\
\text { (Obese) }\end{array}$ & 0.90 & {$[0.84,0.97]$} & 1.05 & {$[0.99,1.12]$} \\
\hline
\end{tabular}


Table 4. Association between peer support and selected physical activity indicators: Results from adjusted three-level logistic regression models with mixed effects; including the peer support x BMI interaction term.

\begin{tabular}{|c|c|c|c|c|}
\hline & \multicolumn{2}{|c|}{ Meet PA Guideline } & \multicolumn{2}{|c|}{$\begin{array}{c}\text { Meet Vigorous PA Frequency } \\
\text { Guidelines }\end{array}$} \\
\hline & OR & $95 \% \mathrm{CI}$ & OR & $95 \% \mathrm{CI}$ \\
\hline Main effects & & & & \\
\hline Peer support & 1.12 & {$[1.08,1.16]$} & 1.16 & {$[1.13,1.20]$} \\
\hline \multicolumn{5}{|l|}{ BMI (ref. = Underweight) } \\
\hline Normal weight & 0.96 & {$[0.92,0.99]$} & 1.22 & {$[1.18,1.26]$} \\
\hline Overweight & 0.72 & {$[0.69,0.76]$} & 1.02 & {$[0.98,1.07]$} \\
\hline Obese & 0.57 & {$[0.53,0.61]$} & 0.81 & {$[0.76,0.86]$} \\
\hline \multicolumn{5}{|l|}{ Interaction effects } \\
\hline $\begin{array}{l}\text { Peer support * BMI } \\
\text { (Normal weight) }\end{array}$ & 1.00 & {$[0.96,1.04]$} & 0.98 & {$[0.95,1.02]$} \\
\hline $\begin{array}{l}\text { Peer support* BMI } \\
\text { (Overweight) }\end{array}$ & 0.99 & {$[0.94,1.04]$} & 1.00 & {$[0.96,1.05]$} \\
\hline Peer support * BMI (Obese) & 1.00 & {$[0.94,1.08]$} & 1.08 & {$[1.02,1.14]$} \\
\hline
\end{tabular}

Table 5. Association between teacher support and selected physical activity indicators: Results from adjusted three-level logistic regression models with mixed effects; including the teacher support $x$ BMI interaction term.

\begin{tabular}{|c|c|c|c|c|}
\hline & \multicolumn{2}{|c|}{ Meet PA Guideline } & \multicolumn{2}{|c|}{$\begin{array}{c}\text { Meet Vigorous PA Frequency } \\
\text { Guidelines }\end{array}$} \\
\hline & OR & $95 \% \mathrm{CI}$ & OR & $95 \% \mathrm{CI}$ \\
\hline \multicolumn{5}{|l|}{ Main effects } \\
\hline Teacher support & 0.92 & {$[0.89,0.95]$} & 0.88 & {$[0.85,0.91]$} \\
\hline \multicolumn{5}{|l|}{ BMI (ref. = Underweight) } \\
\hline Normal & 0.96 & {$[0.93,1.00]$} & 1.21 & {$[1.17,1.26]$} \\
\hline Overweight & 0.72 & {$[0.69,0.76]$} & 1.01 & {$[0.97,1.06]$} \\
\hline Obese & 0.56 & {$[0.52,0.60]$} & 0.78 & {$[0.73,0.83]$} \\
\hline \multicolumn{5}{|l|}{ Interaction effects } \\
\hline $\begin{array}{c}\text { Teacher support * BMI } \\
\text { (normal) }\end{array}$ & 1.05 & {$[1.01,1.09]$} & 1.07 & {$[1.03,1.10]$} \\
\hline $\begin{array}{l}\text { Teacher support * BMI } \\
\text { (overweight) }\end{array}$ & 1.09 & {$[1.03,1.15]$} & 1.08 & {$[1.03,1.13]$} \\
\hline $\begin{array}{c}\text { Teacher support * BMI } \\
\text { (obese) }\end{array}$ & 1.01 & {$[0.94,1.09]$} & 0.98 & {$[0.92,1.04]$} \\
\hline
\end{tabular}

$n=134,148$ adolescents from 37 countries from the 2013/2014 HBSC survey. Data are OR and 95\% confidence intervals. Statistically significant values are indicated in bold. All models were adjusted for sex, age, and SES. $\mathrm{PA}=$ physical activity; $\mathrm{OR}=$ odds ratio; $\mathrm{CI}=$ confidence interval; $\mathrm{BMI}=$ body mass index; $\mathrm{SES}=$ socioeconomic status; HBSC = Health Behaviour in School-aged Children; ref. = reference category.

\section{Discussion}

The study aimed to examine the role of different sources of social support in the relationship of BMI and PA in an international sample of children and adolescents. Threelevel interaction models found a moderating effect of family support on the prevalence of meeting PA guidelines in overweight and obese adolescents, a moderating effect of support from friends on vigorous PA frequency in obese adolescents, and a moderating effect of teacher support on the prevalence of meeting PA guidelines and PA frequency guidelines in overweight and obese adolescents. 
Generally, only $20.0 \%$ of adolescents engaged in PA every day, and $72.7 \%$ of the sample engaged more than twice per week in vigorous PA. This prevalence decreased with increasing age and was lower for girls compared to boys in most countries. In addition, the prevalence was lower for overweight and obese adolescents compared to underweight and normal-weight adolescents and decreased with a decreasing SES score.

Previous research reported similar findings: gender differences in the prevalence of physical inactivity are evident [32]. A pooled analysis with 1.6 million adolescents aged 11-17 years from 146 countries reported insufficient PA for more than four in five schoolaged adolescents. Girls were less active compared to boys across all countries and income groups. Data from representative national and international surveys from 105 countries demonstrate a decreasing prevalence of PA with increasing age and an overall higher prevalence in boys than in girls [33].

Further, we observed gender differences in the perception of social support. Boys reported more support from family; girls perceived more support from friends and teachers (yet this difference was small). As girls usually have a higher tendency for prosocial and cooperative behaviors and engagement in dyadic interactions, this might explain the difference in peer support [34]. Regarding the higher perception of family support in boys, this might be due to the later onset of maturation during adolescence in boys [35]. This is in line with research from Reimers and colleagues who report gender differences in the perception of social support. However, they found that boys perceived more support from family and peers [16]. Yet, another study reported consistently higher levels of social support from family and friends among girls compared to boys [36].

Overall, family support expressed the strongest reduction with increasing age, while peer support remained relatively unaffected and teacher support slightly increased. This might be explained by maturation and adolescents' striving for independence from their parents. Meeus and colleagues reported that support from parents decreased with age, suggesting that this support is experienced in early adolescence before a separation process commences [37]. It is noteworthy that in contrast to family and peer support, teacher support was rather independent of adolescents' SES and BMI. One can assume that this reflects an equal treatment of adolescents by teachers, regardless of their weight or social background. On the other hand, adolescents with higher SES and lower BMI report more family and peer support.

The findings indicate that family support does not increase the prevalence for meeting PA guidelines in adolescents, but slightly increases the prevalence for meeting vigorous PA frequency guidelines. Parents may provide logistical (e.g., transportation) and emotional (e.g., encouragement) support for their child, which influences PA behavior [23]. Suggesting that vigorous PA frequency most likely stems from sporting activities, might explain the results. Either, adolescents who engage in more sporting activities (e.g., in a sports club) might be in higher need of such parental support. Or, as it is not possible to draw conclusions about causation, adolescents who experience this support from their parents are more likely to engage in sporting activities and thus accumulate more vigorous PA. Research supports that adolescents who experience this kind of parental support may engage in more sporting activities [16]. However, this is a surprising finding, as previous research has demonstrated the importance of family support for PA in general [36].

Social support from friends increases both PA and vigorous PA frequency. This is in line with research, which argues that peer support strongly affects PA in adolescence $[16,19,23]$. On the one hand, friends can provide companionship and thus influence PA behavior. Previous research emphasizes the increasing influence of peers with increasing age on activity behavior. However, gender composition and closeness of friendship as well as the friends' behaviors largely impact activity behavior in adolescence [38]. When considering causality issues due to the cross-sectional design, the presented effect might also be rooted in the fact that adolescents who engage, for example, in team sports activities experience more support from friends. As specific activities were not considered within this study, this assumption can neither be confirmed nor negated. 
In contrast to support from family and friends, adolescents who perceive a higher teacher support report lower levels of PA and vigorous PA frequency. Considering the wording of the items used to assess teacher support, it can be observed that this kind of support rather stems from emotional support. According to Brewster and Bowen, characteristics such as warmth and acceptance describe an emotional supportive student-teacher relationship [39]. Consequently, this fails to account for the additional types of support teachers should provide (e.g., providing knowledge and feedback). Classroom teachers prioritize cognitive learning experiences rather than impart an active healthy lifestyle. A health-enhancing education and school environment are only partially implemented in the curricula across Europe, thus teachers are not forced to amend their way of living and teaching.

Whereas, in our model, social support from family did not impact the relationship of vigorous PA frequency and BMI, it had a moderating effect on BMI and overall PA. Generally, meeting PA and vigorous PA frequency guidelines strongly depended on BMI level and gained a positive influence from higher family support. However, the interaction effect from family support was only significant in the relationship for overall PA in the obese and overweight. Consequently, in overweight and obese adolescents the support decreases the likelihood for PA compared to underweight and normal-weight adolescents. Assuming that obese/overweight adolescents are more likely to live in families with obese/overweight parents [40] this moderation might be explained by the modeling effect from parents. However, data do not allow us to draw conclusions about parental activity behavior.

The model including social support from peers reveals no moderation effect in the relationship of PA or vigorous PA frequency and BMI except within the group of obese adolescents. The interaction effect significantly increased the odds of meeting vigorous PA frequency guidelines. This contributes to the evidence from Salvy and colleagues who examined the importance of friends in overweight/obese youth. They argue that peers and friends increase the motivation to be physically active [41].

When teacher support was included in the model, it supported the finding from the three-level logistic regression model that higher teacher support was associated with lower PA and vigorous PA frequency. The interaction effect had a positive influence on PA and vigorous PA frequency within normal-weight and overweight adolescents compared to underweight and obese adolescents. Grounded in attachment theory, teachers may serve as temporary attachment figures in their classrooms and can function as a secure base [42]. Verschueren argues that this may lead to teacher support seeking especially in times of stress [42]. Regarding this, the effects might point at adolescents experiencing more stress or mental health issues and thus require more teacher support. This might also explain the relationship with PA. Generally, PA is related to a reduction in mental health issues [43]. It seems that those who are not able to accumulate a sufficient amount of PA try to counteract stress or mental health issues by seeking support from their teachers. Under this premise, our results suggest that underweight and obese adolescents might require a higher need of emotional support from teachers. Recent reviews from school-based intervention research showed that the impact of teachers on students' daily PA behavior is limited [44]. The question arises whether it is time to rethink the role and education of teachers in Europe and if teachers are capable of creating a supportive (learning) environment that similarly promotes PA among adolescents. Indeed, the curricula of schools and professional teacher education incorporate various educational and pedagogical goals, but in light of the increasing obesity prevalence across Europe, a strategic orientation on health might be worthwhile.

The major strength of our study is the robust analysis of a large dataset, consisting of data derived from validated items and standardized protocols from 37 countries. However, the study's limitations need to be considered. The HBSC study is of cross-sectional design and therefore does not allow conclusions about causation. Furthermore, the study popula- 
tion is restricted to adolescents aged 11-15 years, which limits the generalizability of the findings. Additionally, a high number of values are (systematically) missing.

The self-report data is prone to bias and might have caused an overestimation of activity [45]. Due to the nature of the HBSC study, the assessment of social support does not refer to the domain-specific context of PA. This constitutes an important issue, as measures of social relations, which are specific to PA behaviors, appear to be more predictive than general measures [46]. Further, we need to be aware, that as by their definition both measures for PA used in this study relate to each other and therefore should always be reported in combination. Additionally, during the data collection period for the 2014 HBSC study, the WHO recommended a daily 60 min MVPA [31], whereas these recommendations where adjusted in 2020 to an average of 60 min MVPA per day across the week [1].

\section{Conclusions}

The social environment facilitates the engagement in PA [15,16]. Our study highlights the importance of an intact and trustful family life especially for overweight and obese adolescents. Especially for those, the risk of physical inactivity might decrease when they are a member of an active peer group. Teachers are an important source of emotional support, especially for adolescents who are not sufficiently active. Our findings can assist the development of tailored PA programs depending on the BMI of the target group by providing a rationale for the most relevant source of support. In this context, further research should aim for diverse measures of social support including domain-specific (e.g., support for PA) and non-specific support (e.g., emotional support).

Supplementary Materials: The following tables are available online at https:/ /www.mdpi.com/ article/10.3390/su13136992/s1, Table S1: Description of the total sample and study samples by country. Table S2: Prevalence of at least $60 \mathrm{~min}$ of physical activity per day and at least 2-3 times vigorous physical activity in leisure time by country and sex. Table S3: Prevalence rates of at least 60 min of physical activity per day and at least $2-3$ times vigorous physical activity in leisure time by country and age. Table S4: Prevalence rates of at least $60 \mathrm{~min}$ of physical activity per day and at least 2-3 times vigorous physical activity in leisure time by country and body mass index. Table S5: Prevalence rates of at least $60 \mathrm{~min}$ physical activity per day and at least 2-3 times vigorous physical activity in leisure time by country and socioeconomic status.

Author Contributions: Conceptualization, D.R., A.M. and Y.D.; methodology, D.R., D.J.S.; software, D.J.S., validation, J.G., B.M., M.P.; formal analysis, D.J.S., D.R.; investigation, D.R., D.J.S.; writingoriginal draft preparation, D.R., D.J.S.; writing—review and editing, D.R., D.J.S., A.M., M.P., B.M., J.G., S.P., Y.D.; supervision, A.M., S.P., Y.D.; project administration, A.M., Y.D., S.P. All authors have read and agreed to the published version of the manuscript.

Funding: This work was supported by the ERASMUS+ Sport program [grant number 613324-EPP-12019-1-PT-SPO-SCP]. The content of this document represents the views of the authors only and is their sole responsibility; it cannot be considered to reflect the views of the Education, Audiovisual and Culture Executive Agency (EACEA) of the European Commission or any other body of the European Union. The European Commission and the Agency do not accept any responsibility for use that may be made of the information it contains.

Institutional Review Board Statement: Authors performed a secondary analysis of the HBSC data. For details on ethical review and approval of the HBSC study see http:/ / www.hbsc.org/methods / (accessed on 24 March 2021).

Informed Consent Statement: Informed consent was obtained during the course of the HBSC study from all subjects involved in the study.

Data Availability Statement: The data used in this study are available at http:/ / www.uib.no/en/ hbscdata39357 (accessed on 24 March 2021).

Acknowledgments: HBSC is an international study carried out in collaboration with WHO/EURO. For details, see http:/ / www.hbsc.org (accessed on 24 March 2021). 
Conflicts of Interest: The authors declare no conflict of interest.

\section{References}

1. WHO. WHO Guidelines on Physical Activity and Sedentary Behaviour; World Health Organization: Geneva, Switzerland, 2020.

2. Poitras, V.J.; Gray, C.E.; Borghese, M.M.; Carson, V.; Chaput, J.-P.; Janssen, I.; Katzmarzyk, P.T.; Pate, R.R.; Gorber, S.C.; Kho, M.E.; et al. Systematic review of the relationships between objectively measured physical activity and health indicators in school-aged children and youth. Appl. Physiol. Nutr. Metab. 2016, 41, 197-239. [CrossRef]

3. Marques, A.; Demetriou, Y.; Tesler, R.; Gouveia, É.R.; Peralta, M.; de Matos, M.G. Healthy Lifestyle in Children and Adolescents and Its Association with Subjective Health Complaints: Findings from 37 Countries and Regions from the HBSC Study. Int. J. Environ. Res. Public Health 2019, 16, 3292. [CrossRef] [PubMed]

4. Janssen, I.; LeBlanc, A.G. Systematic review of the health benefits of physical activity and fitness in school-aged children and youth. Int. J. Behav. Nutr. Phys. Act. 2010, 7, 40. [CrossRef]

5. Telama, R.; Yang, X.; Leskinen, E.; Kankaanpää, A.; Hirvensalo, M.; Tammelin, T.; Viikari, J.S.; Raitakari, O.T. Tracking of physical activity from early childhood through youth into adulthood. Med. Sci. Sports Exerc. 2014, 46, 955-962. [CrossRef] [PubMed]

6. Hallal, P.C.; Victora, C.G.; Azevedo, M.R.; Wells, J.C.K. Adolescent Physical Activity and Health. Sports Med. 2006, 36, 1019-1030. [CrossRef] [PubMed]

7. WHO. Adolescent Obesity and Related Behaviours: Trends and Inequalities in the WHO European Region, 2002-2014; WHO Regional Office for Europe: Copenhagen, Denmark, 2017.

8. NCD Risk Factor Collaboration (NCD-RisC). Worldwide trends in body-mass index, underweight, overweight, and obesity from 1975 to 2016: A pooled analysis of 2416 population-based measurement studies in 128.9 million children, adolescents, and adults. Lancet 2017, 390, 2627-2642. [CrossRef]

9. Ortega, F.B.; Ruiz, J.R.; Castillo, M.J. Physical activity, physical fitness, and overweight in children and adolescents: Evidence from epidemiologic studies. Endocrinol. Nutr. 2013, 60, 458-469. [CrossRef]

10. Ekelund, U.; Sardinha, L.B.; Anderssen, S.A.; Harro, M.; Franks, P.W.; Brage, S.; Cooper, A.R.; Andersen, L.B.; Riddoch, C.; Froberg, K. Associations between objectively assessed physical activity and indicators of body fatness in 9- to 10-y-old European children: A population-based study from 4 distinct regions in Europe (the European Youth Heart Study). Am. J. Clin. Nutr. 2004, 80, 584-590. [CrossRef]

11. Butte, N.F.; Puyau, M.R.; Adolph, A.L.; Vohra, F.A.; Zakeri, I. Physical Activity in Nonoverweight and Overweight Hispanic Children and Adolescents. Med. Sci. Sports Exerc. 2007, 39, 1257-1266. [CrossRef]

12. Elmesmari, R.; Martin, A.; Reilly, J.J.; Paton, J.Y. Comparison of accelerometer measured levels of physical activity and sedentary time between obese and non-obese children and adolescents: A systematic review. BMC Pediatrics 2018, 18, 106. [CrossRef]

13. Jiménez-Pavón, D.; Kelly, J.; Reilly, J.J. Associations between objectively measured habitual physical activity and adiposity in children and adolescents: Systematic review. Int. J. Pediatric Obes. 2010, 5, 3-18. [CrossRef] [PubMed]

14. Biro, F.M.; Wien, M. Childhood obesity and adult morbidities. Am. J. Clin. Nutr. 2010, 91, 1499-1505. [CrossRef]

15. Sallis, J.F.; Cervero, R.B.; Ascher, W.; Henderson, K.A.; Kraft, M.K.; Kerr, J. An ecological approach to creating active living communities. Annu. Rev. Public Health 2006, 27, 297-322. [CrossRef]

16. Reimers, A.K.; Schmidt, S.C.E.; Demetriou, Y.; Marzi, I.; Woll, A. Parental and peer support and modelling in relation to domain-specific physical activity participation in boys and girls from Germany. PLoS ONE 2019, 14, e0223928. [CrossRef]

17. Bauman, A.E.; Reis, R.S.; Sallis, J.F.; Wells, J.C.; Loos, R.J.F.; Martin, B.W. Correlates of physical activity: Why are some people physically active and others not? Lancet 2012, 380, 258-271. [CrossRef]

18. Craggs, C.; Corder, K.; van Sluijs, E.M.F.; Griffin, S.J. Determinants of Change in Physical Activity in Children and Adolescents: A Systematic Review. Am. J. Prev. Med. 2011, 40, 645-658. [CrossRef]

19. Duncan, S.C.; Duncan, T.E.; Strycker, L.A. Sources and Types of Social Support in Youth Physical Activity. Health Psychol. 2005, 24, 3-10. [CrossRef]

20. Sallis, J.F.; Taylor, W.C.; Dowda, M.; Freedson, P.S.; Pate, R.R. Correlates of Vigorous Physical Activity for Children in Grades 1 through 12: Comparing Parent-Reported and Objectively Measured Physical Activity. Pediatric Exerc. Sci. 2002, 14, 30. [CrossRef]

21. Van der Horst, K.; Paw, M.J.C.A.; Twisk, J.W.; Van Mechelen, W. A brief review on correlates of physical activity and sedentariness in youth. Med. Sci. Sports Exerc. 2007, 39, 1241-1250. [CrossRef] [PubMed]

22. Badura, P.; Hamrik, Z.; Dierckens, M.; Gobina, I.; Malinowska-Cieślik, M.; Furstova, J.; Kopcakova, J.; Pickett, W. After the bell: Adolescents' organised leisure-time activities and well-being in the context of social and socioeconomic inequalities. J. Epidemiol. Community Health 2021, 75, 628-636. [CrossRef] [PubMed]

23. Gill, M.; Chan-Golston, A.M.; Rice, L.N.; Roth, S.E.; Crespi, C.M.; Cole, B.L.; Koniak-Griffin, D.; Prelip, M.L. Correlates of Social Support and its Association With Physical Activity Among Young Adolescents. Health Educ. Behav. 2018, 45, 207-216. [CrossRef]

24. De Bourdeaudhuij, I.; Lefevre, J.; Deforche, B.; Wijndaele, K.; Matton, L.; Philippaerts, R. Physical Activity and Psychosocial Correlates in Normal Weight and Overweight 11 to 19 Year Olds. Obes. Res. 2005, 13, 1097-1105. [CrossRef] [PubMed]

25. Blanchard, C.M.; McGannon, K.R.; Spence, J.C.; Rhodes, R.E.; Nehl, E.; Baker, F.; Bostwick, J. Social ecological correlates of physical activity in normal weight, overweight, and obese individuals. Int. J. Obes. 2005, 29, 720-726. [CrossRef] 
26. Currie, C.; Inchley, J.; Molcho, M.; Lenzi, M.; Veselska, Z.; Wild, F. Health Behaviour in School-Aged Children (HBSC) Study Protocol: Background, Methodology and Mandatory Items for the 2013/14 Survey; Child and Adolescent Health Research Unit (CAHRU): St Andrews, UK, 2014.

27. Boyce, W.; Torsheim, T.; Currie, C.; Zambon, A. The Family Affluence Scale as a Measure of National Wealth: Validation of an Adolescent Self-Report Measure. Soc. Indic. Res. 2006, 78, 473-487. [CrossRef]

28. WHO. BMI-for-Age (5-19 Years). Available online: https://www.who.int/toolkits/growth-reference-data-for-5to19-years/ indicators /bmi-for-age (accessed on 12 April 2021).

29. de Onis, M.; Onyango, A.W.; Borghi, E.; Siyam, A.; Nishida, C.; Siekmann, J. Development of a WHO growth reference for school-aged children and adolescents. Bull. World Health Organ. 2007, 85, 660-667. [CrossRef] [PubMed]

30. Zimet, G.D.; Dahlem, N.W.; Zimet, S.G.; Farley, G.K. The Multidimensional Scale of Perceived Social Support. J. Personal. Assess. 1988, 52, 30-41. [CrossRef]

31. WHO. Global Recommendations on Physical Activity for Health; 9241599979; World Health Organization: Geneva, Switzerland, 2010.

32. Guthold, R.; Stevens, G.A.; Riley, L.M.; Bull, F.C. Global trends in insufficient physical activity among adolescents: A pooled analysis of 298 population-based surveys with 1.6 million participants. Lancet Child Adolesc. Health 2020, 4, 23-35. [CrossRef]

33. Marques, A.; Henriques-Neto, D.; Peralta, M.; Martins, J.; Demetriou, Y.; Schönbach, D.M.I.; Gaspar de Matos, M. Prevalence of Physical Activity among Adolescents from 105 Low, Middle, and High-Income Countries. Int. J. Environ. Res. Public Health 2020, 17, 3145. [CrossRef] [PubMed]

34. Rose, A.J.; Rudolph, K.D. A review of sex differences in peer relationship processes: Potential trade-offs for the emotional and behavioral development of girls and boys. Psychol. Bull. 2006, 132, 98-131. [CrossRef] [PubMed]

35. Klimstra, T.A.; Hale Iii, W.W.; Raaijmakers, Q.A.W.; Branje, S.J.T.; Meeus, W.H.J. Maturation of personality in adolescence. J. Personal. Soc. Psychol. 2009, 96, 898-912. [CrossRef]

36. Khan, S.R.; Uddin, R.; Mandic, S.; Khan, A. Parental and Peer Support are Associated with Physical Activity in Adolescents: Evidence from 74 Countries. Int. J. Environ. Res. Public Health 2020, 17, 4435. [CrossRef] [PubMed]

37. Meeus, W.; Iedema, J.; Maassen, G.; Engels, R. Separation-individuation revisited: On the interplay of parent-adolescent relations, identity and emotional adjustment in adolescence. J. Adolesc. 2005, 28, 89-106. [CrossRef] [PubMed]

38. Chung, S.J.; Ersig, A.L.; McCarthy, A.M. The Influence of Peers on Diet and Exercise Among Adolescents: A Systematic Review. J. Pediatric Nurs. Nurs. Care Child. Fam. 2017, 36, 44-56. [CrossRef]

39. Brewster, A.B.; Bowen, G.L. Teacher Support and the School Engagement of Latino Middle and High School Students at Risk of School Failure. Child Adolesc. Soc. Work J. 2004, 21, 47-67. [CrossRef]

40. Bahreynian, M.; Qorbani, M.; Khaniabadi, B.M.; Motlagh, M.E.; Safari, O.; Asayesh, H.; Kelishadi, R. Association between Obesity and Parental Weight Status in Children and Adolescents. J. Clin. Res. Pediatr. Endocrinol. 2017, 9, 111-117. [CrossRef]

41. Salvy, S.-J.; Bowker, J.C.; Germeroth, L.; Barkley, J. Influence of peers and friends on overweight/obese youths' physical activity. Exerc. Sport Sci. Rev. 2012, 40, 127-132. [CrossRef]

42. Verschueren, K. Middle Childhood Teacher-Child Relationships: Insights From an Attachment Perspective and Remaining Challenges. New Dir. Child Adolesc. Dev. 2015, 2015, 77-91. [CrossRef]

43. Biddle, S.J.; Asare, M. Physical activity and mental health in children and adolescents: A review of reviews. Br. J. Sports Med. 2011, 45, 886-895. [CrossRef] [PubMed]

44. Yuksel, H.S.; Şahin, F.N.; Maksimovic, N.; Drid, P.; Bianco, A. School-Based Intervention Programs for Preventing Obesity and Promoting Physical Activity and Fitness: A Systematic Review. Int. J. Environ. Res. Public Health 2020, 17, 347. [CrossRef]

45. Hagströmer, M.; Bergman, P.; De Bourdeaudhuij, I.; Ortega, F.B.; Ruiz, J.R.; Manios, Y.; Rey-López, J.P.; Phillipp, K.; von Berlepsch, J.; Sjöström, M.; et al. Concurrent validity of a modified version of the International Physical Activity Questionnaire (IPAQ-A) in European adolescents: The HELENA Study. Int. J. Obes. 2008, 32, S42-S48. [CrossRef] [PubMed]

46. Tay, L.; Tan, K.; Diener, E.; Gonzalez, E. Social Relations, Health Behaviors, and Health Outcomes: A Survey and Synthesis. Appl. Psychol. Health Well Being 2013, 5, 28-78. [CrossRef] [PubMed] 